\title{
Relapsing Polychondritis with Multifocal Neurological Abnormalities
}

\author{
J. Willis, E.A. Atack, G. Kraag
}

ABSTRACT: A sixty-five year old woman developed relapsing polychondritis with three of the diagnostic criteria established by McAdam et al. (1976), namely bilateral auricular chondritis, ocular inflammation and both cochlear and vestibular dysfunction. Many authors have mentioned other neurological symptoms including unilateral facial weakness. This patient is the first case described with bilateral facial weakness and cerebral manifestations.

Relapsing polychondritis, an uncommon, recurrent, inflammatory disorder affecting the cartilaginous tissues of the body (JakschWartenhorst, 1923; Herman, 1981), is regularly associated with audiovestibular dysfunction (Bollet et al., 1969; Cody et al., 1971; McAdam et al., 1976; Ridgway et al., 1979). McAdam et al. (1976) and Ridgway et al. (1979) have mentioned other neurological symptoms including unilateral facial weakness. We recently observed a patient with polychondritis and multifocal neurological abnormalities. We believe this is the first case described with bilateral facial nerve palsy and with cerebral manifestations.

RÉSUMÉ: Une dame de soixante-cinq ans a développé une polychondrite récidivante avec trois des critères diagnostics établis par McAdam et Coll (1976), soit une chondrite auriculaire bilatérale, une inflammation oculaire et une dysfonction à la fois cochléaire et véstibulaire. Plusieurs auteurs ont signalé d'autres symptômes neurologiques incluant une parésie faciale unilatérale. Cette observation d'une parésie faciale bilatérale avec manifestations cérébrales est la première à être signalée.

Can. J. Neurol. Sci. 1984; 11:402-404

\section{Case RePORT}

A 64 year old housewife with diabetes mellitus treated for two years with tolbutamide, $300 \mathrm{mgm}$ b.i.d. presented in September, 1981 with a one week history of fever and a painful right ear. She was treated with an oral cephalosporin, but was admitted two days later to a community hospital because of persistent fever and pain. The cephalosporin was replaced by parenteral ampicillin for suspected mastoiditis. She continued to complain of pain in both ears, difficulty hearing, irritation in both eyes, fatigue and low back pain. Following the abrupt onset of right facial weakness she was referred to the neurological service at the Ottawa Civic Hospital.

On admission she had bilateral conjunctivitis and right facial weakness associated with Bell's phenomenon. Taste was reported to be normal. Other than a reduction in hearing of whispered sound in the right ear and tenderness of both external auditory meati the remainder of the examination was normal.

Laboratory findings revealed a leukocytosis of $16,000 \mathrm{cells} / \mathrm{mm}^{3}$ and an elevated erythrocyte sedimentation rate of $49 \mathrm{~mm} / \mathrm{hr}$. Her diabetes was not controlled with a plasma glucose of 297 , urine +3 for glucose and a small amount of ketones. Serum electrolytes, tests of renal and liver function were normal.

Within twenty-four hours a left facial nerve palsy developed. Her hearing impairment increased, she became confused and began having paranoid delusions with fever as high as $40^{\circ} \mathrm{C}$ orally. Cultures of the conjunctiva, ears, blood, urine and CSF were negative. An electroencephalogram showed a diffuse slow wave abnormality. Roentgenograms of the skull, tomograms of the internal auditory meati, and computerized tomography of the head were all normal. Rheumatoid factor was 1:40 and antinuclear antibody was detected 1:80 with a specked pattern. Serum protein electrophoresis demonstrated hypergammaglobulinemia. Cryoglobulins were negative. Audiometric studies revealed bilateral hearing loss with the left side more severely affected than the right. The right ear was 30 decibels at $250 \mathrm{~Hz}$ and 90 decibels at $8000 \mathrm{hz}$. The left ear was 90 decibels at $250 \mathrm{~Hz}$ and 100 decibels at $8000 \mathrm{hz}$.

At the time an allergic conjunctivitis and Ramsay-Hunt Syndrome were considered in the differential diagnosis but no specific allergen was identified and at no time did herpetic lesions develop.

From the Division of Neurology, Department of Medicine, Ottawa Civic Hospital and the University of Ottawa Medical School.

Received January 25, 1983. Accepted in revised form May 10, 1984.

Reprints requests to: Dr. E.A. Atack, Chief, Division of Neurology, Ottawa Civic Hospital D-7, Ottawa, Canada KIY 4E9 
After two weeks in hospital, with control of her diabetes and a course of parenteral penicillin and gentamicin, there was no improvement in her condition. During this time she continued to experience paranoid delusions and at times required physical restraints. Finally with the development of abrupt painful cauliflower swelling and violaceous erythema of both external ears (Figure 1) and slight tenderness of the thyroid cartilage, a diagnosis of relapsing polychondritis was made. Because the clinical picture was typical of relapsing polychondritis, a biopsy of the external ear was not taken and a course of prednisone 40 mgm daily in divided doses was started.

Within a few days there was resolution of fever, reduction of the swelling in both ears and of the redness of both eyes. Repeat audiometric studies showed marked improvement from that taken earlier. Defects in hearing remained however, particularly in the left ear. The right was $10 \mathrm{~dB}$ at $250 \mathrm{~Hz}$ and $50 \mathrm{~dB}$ at $8000 \mathrm{~Hz}$. The left ear was $60 \mathrm{~dB}$ at $250 \mathrm{~Hz}$ and also $60 \mathrm{~dB}$ at $8000 \mathrm{~Hz}$. After a further two weeks some improvement of her facial weakness, particularly on the right side and gradual clearing of her confusion and delusions had occurred.

On follow up examination in August 1982 there was no evidence of confusion or paranoid ideation and only a mild left facial weakness remained. The patient remained partly deaf in the left ear with some impairment of balance. She continued on a low dose of prednisone.

\section{Discussion}

Relapsing polychondritis, an episodic systematic disorder, is characterized by widespread inflammatory lesions involving cartilaginous structures and connective tissues of the respira-

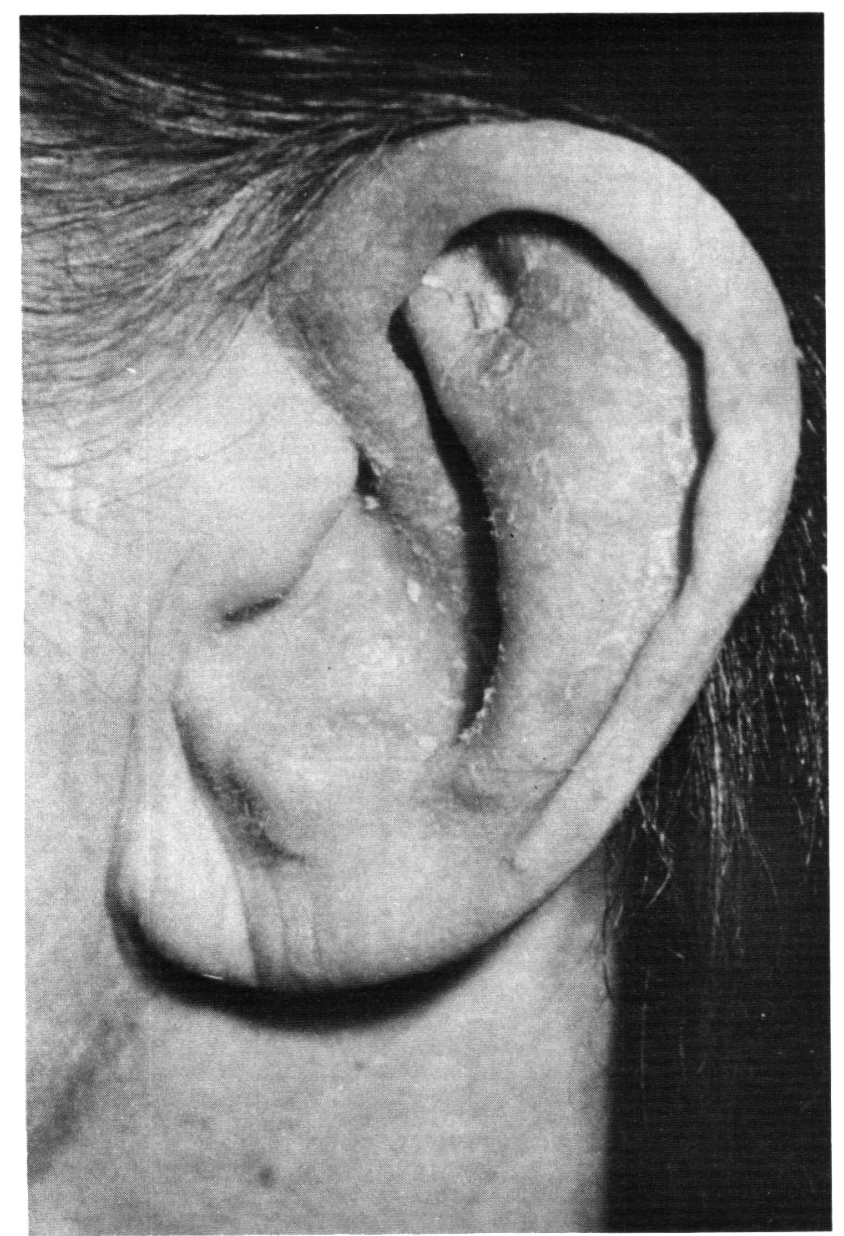

Figure l - Left external ear of patient, showing cauliflower swelling and violaceous erythema characteristic of relapsing polychondritis. tory tract, the cardiovascular system, and eyes and ears. (JakschWartenhorst, 1923; Bollet et al., 1969; Cody et al., 1971; McAdam et al., 1976; Ridgway et al., 1979; Herman, 1981).

The pathogenesis of the disorder is not known and there are no specific laboratory tests (Herman, 1981). However, it is presumed that the lesion is an immunological injury to matrix and mucopolysaccharide.

According to proposed criteria established by McAdam et al. (1976) a definitive diagnosis can be made with three or more of the following clinical features: bilateral auricular chondritis, nonerosive seronegative inflammatory polyarthritis, nasal chondritis, ocular inflammation, respiratory tract chondritis, cochlear and/or vestibular dysfunction. In a clinically obvious case biopsy was deemed unnecessary for confirmation of the diagnosis.

The condition is more common than generally appreciated with approximately 250 cases having been reported. More subtle modes of presentation can cause difficulty in diagnosis and delay effective therapy.

It has been postulated that an arteritis of the internal auditory artery or its vestibular branch results in episodic vertigo, ataxia, and nausea (McAdam et al., 1976). A similar mechanism may well be responsible for the facial nerve palsy. As many as $30 \%$ of patients develop other autoimmune disease processes at one time or another (Herman, 1981). It is not certain whether neurological problems are strictly attributable to relapsing polychondritis or to an associated systemic vasculitis. Involvement of neurological structures remote from cartilaginous tissues must necessarily be related to the latter.

A review of 165 cases from the literature reveals that neurological problems have been noted in the following approximate percentages: reduced hearing $39 \%$, vertigo $25 \%$, headaches $22 \%$, tinnitus $18 \%$, nystagmus $9 \%$, paresthesia $5 \%$, optic neuritis $4 \%$, diplopia 3.5\%, facial weakness $2 \%$ (Kaye et al., 1964; Dolan et al., 1966; Hughes et al., 1972; McAdam et al., 1976; Ridgway et al., 1979).

Confusion, disordered thinking, and an abnormal electroencephalogram were prominent manifestations of our patient's illness. In previous cases, encephalopathic features have not been described. Our patient showed a beneficial response to a medium dose of prednisone with initial fairly rapid improvement in the suspected cerebral arteritis and resolution of facial weakness.

The possibility that the bilateral facial weakness was related to her diabetes was considered. Since it developed with the acute phase of the auricular chondritis and gradually cleared along with the other manifestations of the patient's relapsing polychondritis we believe it was more likely related to this entity.

Although thorough ophthalmologic examination was performed and no keratitis found, Cogan's syndrome could have explained the associated eye signs with the audiovestibular dysfunction (Bicknell and Holland, 1978), but the patient's age, the auricular chondritis and the marked improvement in hearing would be inconsistent with this diagnosis. However, Cogan's syndrome and relapsing polychondritis do have overlapping features in their clinical presentation.

The course of relapsing polychondritis and the response to treatment can be quite variable. Many cases do well on steroid therapy as did our case. Good results have also been reported with dapsone (Barranco et al., 1976; Martin et al., 1976) but 
occasionally more potent and potentially more toxic immunosuppressant therapy is required (Herman, 1981).

\section{REFERENCES}

Barranco V, Minor D, Solomon H (1976) Treatment of R.P. with Dapsone. Arch. Dermatol. 112: 1286-1288.

Bicknell JM, Holland JV (1978) Neurologic manifestations of Cogan syndrome. Neurology 28: 278-281.

Bollet A, Smith J, Mushet G, Austin F (1969) Arthritis, Deafness and Saddle Nose. J. Med. Assoc. G.A., 58: 315.

Cody D, Jones D (1971) Relapsing polychondritis: Audio-Vestibular Manifestations, Laryngoscope, 81: 1208.

Dolan D, Lemmon G, Teitelbaum S (1966) Relapsing Polychondritis Analytical Literature Review Am. J. Med. 41: 285.
Herman J (1981) Relapsing Polychondritis Textbook of Rheumatology, William N. Kelly et al., Vol. 2: 1500-8.

Hughes R, Berry C, Seifert M (1972) Relapsing Polychondritis: Clinicopathological Study and Literature Review. Q.J. Med., 61: 363.

Jacksch-Wartenhorst J, (1923) Polychondropathia. Arch. Intern. Med. 6: 93-100.

Kaye R, Jones D (1964) R.P. Clinical and Pathological Features in 14 cases. Ann. Intern. Med., 60: 653.

Martin J, Roenick H, Lynch W (1976) R.P. Treated with Dapsone. Arch. Dermatol. 112: 1272-1274.

McAdam L, O'Hanlan M, Bluestone R, Pearson C (1976) Relapsing Polychondritis: Prospective Study of 23 Patients. Medicine, 55: 193-215.

Ridgway H, Hansotia D, Schorr W (1979) Relapsing Polychondritis: Unusual Neurological Findings and Therapeutic Efficacy of Dapsone. Arch. Dermatol., 115: 43-45. 